\title{
Mechanism for the Formation of Arc-Shaped Cloud Lines over the Tropical Oceans
}

\author{
Claudia Christine STEPHAN ${ }^{\mathrm{a}}$ \\ ${ }^{\text {a }}$ Max Planck Institute for Meteorology, Hamburg, Germany
}

(Manuscript received 1 May 2020, in final form 24 December 2020)

\begin{abstract}
Satellite images frequently show mesoscale arc-shaped cloud lines with a spacing of several tens of kilometers. These clouds form in a shallow mixed boundary layer in locations where the near-surface horizontal wind speed exceeds $\sim 7 \mathrm{~m} \mathrm{~s}^{-1}$. Unlike other mesoscale cloud line phenomena, such as horizontal convective rolls, these cloud lines do not align with the wind direction but form at large oblique angles to the near-surface wind. A particularly distinct event of this pattern developed on 31 January 2020 over the western tropical Atlantic Ocean. Radiosonde soundings are available for this time and location, allowing a detailed analysis. By comparing observations with theoretical predictions that are based on Jeffreys's drag-instability mechanism, it is shown that drag-instability waves may contribute to the formation of this cloud pattern. The theory is formulated in only two dimensions and predicts that wavelike horizontal wind perturbations of this wavelength can grow, because they modulate the surface friction in a way that reinforces the perturbations. The theoretical horizontal wavelengths of $40-80 \mathrm{~km}$ agree with the observations. Streamlines from the ERA5 reanalysis show that the directional change of the near-surface wind is likely to contribute to the arc shape but that a radial propagation of an initial instability is also required to explain the strong curvature. Moreover, ERA5 winds suggest that other known explanations for the formation of cloud lines are unlikely to apply in the case studied here.
\end{abstract}

KEYWORDS: Atlantic Ocean; Tropics; Convective clouds; Gravity waves; Instability; Boundary layer

\section{Introduction}

The planetary boundary layer hosts a wide variety of shallow convective cloud patterns. Some are irregular, although they may nevertheless be classified based on their overall appearance (Stevens et al. 2020). Others develop into highly regular linear or cellular shapes (Atkinson and Wu Zhang 1996). Cellular convection occurs as either open or closed circulation systems (Agee 1987). Linear mesoscale convection forms quasi-two-dimensional elongated structures that are often referred to as bands, lines or rolls. A wide range of processes has been identified to explain their formation (Young et al. 2002). For example, along-stream streaks of clouds can form due to shear-driven up- and downdrafts (Khanna and Brasseur 1998). Shallow rolls oriented along the wind direction can also be forced by a combination of shear- and buoyancy-driven planetary boundary layer flow (Moeng and Sullivan 1994). Horizontal convective rolls are counterrotating vortex rolls that are nearly aligned with the mean wind of the convective boundary layer and form due to shear or dynamic inflection point instabilities of the Ekman layer (Brown 1980). Cloud lines may develop $Y$-shaped branches, so-called dendritic patterns, when their secondary circulation interacts with the surrounding shear and stability profiles (Nicholls and Young 2007).

Fewer examples exist for cloud lines with an orientation perpendicular to the wind. These can occur, for instance, when boundary layer thermals generate gravity waves that propagate within the overlying layer and interact with the boundary layer thermals (Clark et al. 1986; Balaji and Clark 1988; Hauf and Clark 1989; Lane and Clark 2002). Banded structures arise

Corresponding author: Claudia Christine Stephan, claudia.stephan@mpimet.mpg.de when the shear inside the boundary layer is at a $90^{\circ}$ angle with the shear near the cloud top. In this case the resulting banded structures are parallel to the phase lines of the gravity waves and perpendicular to the wind near the cloud tops. However, the bands are still parallel to the near-surface wind (Balaji and Clark 1988).

In this study we present a mechanism to explain the formation of arc-shaped cloud lines at large oblique angles to the near-surface wind. Unlike Balaji and Clark (1988)'s mechanism, it is mainly the mean wind in the well-mixed boundary layer that determines the orientation of the cloud lines. The mechanism is not new; it was already described by Jeffreys (1925) almost a century ago. Nevertheless, to our knowledge, the connection to the frequently occurring phenomenon of cloud lines perpendicular to the near-surface wind has not been established. These cloud lines resemble ripples that form on water-covered pavement during heavy rainfall. Their morphology is very different from the wind-perpendicular lines studied by Balaji and Clark (1988). The arc-shaped patterns discussed here have sharper cloud lines with a greater intercloud spacing, cover larger areas and often persist for more than a day.

The original article by Jeffreys (1925) describes water flowing down an inclined channel. In this case the gravitational acceleration is balanced by surface drag, which is felt by the whole layer of fluid due to turbulent mixing. Instabilities are able to develop because the drag is proportional to the square of the flow velocity. This allows for nonlinear feedbacks between the drag, the flow velocity and the associated convergence or divergence of fluid. Wave crests appear in the form of a periodic buildup of water when the flow-speed-modulated drag force positively reinforces the perturbations in fluid depth. Chimonas (1993) generalized this idea by replacing the gravitational acceleration on slanted terrain with the 
acceleration due to pressure gradients over flat terrain [see Figs. 1 and 2 of Chimonas (1993) for schematics of the force balance]. The turbulent sheet of water is replaced by a turbulent atmospheric boundary layer. In either system instabilities are able to build when waves modulate the surface drag in a way that causes a positive reinforcement. Chimonas (1994) applied the theory to observed phenomena, including a lee wave, the morning glory over Australia, and a storm wave, to explain their amplitudes or longevity, even though their primary forcing mechanisms are not the drag instability. In this study we suggest that the drag-instability mechanism described by Chimonas (1993) is the primary driver of arc-shaped cloud lines that are oriented approximately perpendicular to the wind.

Observational data are introduced in section 2. In section 3 we summarize the drag-instability mechanism and derive the equations for predicting characteristic properties of the cloud pattern. Section 4 examines an observed case of arc-shaped clouds and compares it with theoretical predictions. Section 5 presents the conclusions.

\section{Observations}

Images from the Geostationary Operational Environmental Satellite-East (GOES-E), which is centered on $75.2^{\circ} \mathrm{W}$, serve to introduce the arc-shaped cloud line pattern. These visible $(0.64 \mu \mathrm{m}$; Band 2$)$ and infrared $(10.3 \mu \mathrm{m}$; Band 13$)$ images of the GOES-E Advanced Baseline Imager (ABI; Schmit et al. 2017) are provided by NASA's Worldview application (https:// worldview.earthdata.nasa.gov). The temporal resolution is 10 min, covering 6 August 2019 to present. This study uses data from 1 January to 1 February 2020. The sensor resolution of Band 2 is $0.5 \mathrm{~km}$, and the resolution in Worldview is $1 \mathrm{~km}$. Band 13 has a sensor resolution of $2 \mathrm{~km}$ and is also displayed at $2 \mathrm{~km}$ in Worldview.

For occurrences of the arc-shaped cloud line pattern we analyze estimates of $10-\mathrm{m}$ wind speed over the ocean, taken from the Advanced Microwave Scanning Radiometer 2 (AMSR2) on board the Global Change Observation MissionWater 1 (GCOM-W1) satellite with a sensor resolution of $5 \mathrm{~km}$. The data are obtained by correlating $10-\mathrm{m}$ wind speeds with the measured ocean surface roughness (Kummerow et al. 2015). Daily data are provided for day and night separately and are made available by NASA's Worldview application covering 7 June 2015 to present.

For information on the cloud-top level, we study the cloud-top pressure product images of the GOES-E ABI for both daytime and nighttime conditions (NOAA 2018), which are distributed by the Langley SATCORPS group of NASA at spatial and temporal resolutions of $2 \mathrm{~km}$ and $1 \mathrm{~min}$, respectively.

We analyze wind, temperature, and relative humidity profiles of atmospheric soundings with a vertical resolution of $10 \mathrm{~m}$ collected on 31 January and 1 February 2020, during the campaign Elucidating the Role of Clouds-Circulation Coupling in Climate (EUREC ${ }^{4}$ A; Stephan et al. 2020). The full dataset covers 8 January-19 February 2020 and consists of about 800 radiosonde ascents, with descents recorded for $82 \%$ of them. During normal operations, radiosondes were launched every
$4 \mathrm{~h}$ from five platforms that include the Barbados Cloud Observatory and four ships that moved inside $6^{\circ}-16^{\circ} \mathrm{N}, 51^{\circ}-$ $60^{\circ} \mathrm{W}$. The data coverage differs from platform to platform.

In addition, we use horizontal winds from the ERA5 reanalysis (ERA5 2017), which is publicly available from the ECMWF. At the time of this publication, hourly ERA5 data are available on a 30-km horizontal grid from 1979 to within 5 days of real time. The vertical coordinate consists of 137 hybrid sigma/pressure levels from the surface up to a height of $80 \mathrm{~km}$. This study relies on data between the surface and $1500 \mathrm{~m}$ on 31 January 2020 at 0900,1200 , and 1500 UTC within $8^{\circ}-13^{\circ} \mathrm{N}, 51^{\circ}-46^{\circ} \mathrm{W}$.

\section{Drag-instability mechanism}

Following Chimonas (1993) we now derive the equation system that describes the drag-instability mechanism in the two-dimensional space of the horizontal wave-propagation direction and height. We denote by $x$ and $z$ the horizontal and vertical coordinates, respectively. Homogeneity is assumed along the third spatial dimension. The horizontal velocity component along the $x$ dimension is $u$, and $w$ is the vertical velocity component.

We use a two-layer model of irrotational (no Coriolis force), incompressible fluids, which describes a turbulent surface layer that is separated by a capping inversion from an overlaying semi-infinite layer with a stratification that is either neutral or stable, and with constant wind speed.

The continuity equation in the lower layer is $\partial u / \partial x+\partial w / \partial z=0$. Integration from $z=0$ to $z=h$ with the condition $w(0)=0$ gives $w(h)=-h(\partial u / \partial x)$ if we assume that $u$ does not depend on $z$, which is a valid approximation as long as the horizontal wavelength associated with variability in $u$ is long relative to $h$ (Jeffreys 1925). Since $w(h)=\partial h / \partial t+u(\partial h / \partial x)$, we can express $\partial h / \partial t=-\partial(u h) / \partial x$. Linearization around a mean state $\left(h_{0}, u_{0}, p_{0}\right)$ with wavelike perturbations $\sim \exp [i(\omega t-k x)]$, denoted by a prime, leads to

$$
\left(\frac{\partial}{\partial t}+u_{0} \frac{\partial}{\partial x}\right) h^{\prime}=-h_{0} \frac{\partial u^{\prime}}{\partial x} .
$$

For $h^{\prime} \sim \exp [i(\omega t-k x)]$ this yields

$$
\frac{\partial u^{\prime}}{\partial x}=-\frac{h^{\prime}}{h_{0}}\left(i k c-i k u_{0}\right),
$$

where the phase speed $c=\omega / k$.

The momentum balance for the constant background flow in the boundary layer is

$$
\frac{\partial p_{0}}{\partial x}+d_{0} \rho_{0}=0
$$

for the steady state, where $d_{0}$ is the drag force per unit mass. An assumption of central importance to the drag-instability mechanism is that the aerodynamic surface friction is approximately proportional to the square of the wind speed. Moreover, it is assumed that due to turbulent mixing the drag is felt by the entire boundary layer of depth $h_{0}$ and constant density $\rho_{0}$, such that 


\section{(a) GOES-E vis, Jan 31 1800UTC}

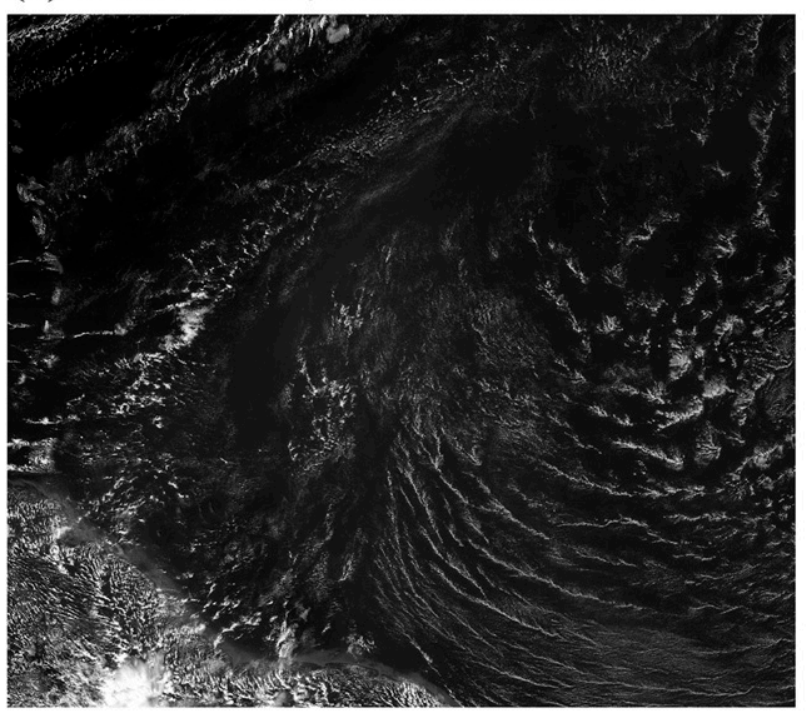

\section{(c) Surface wind speed, Feb 1 night+day}

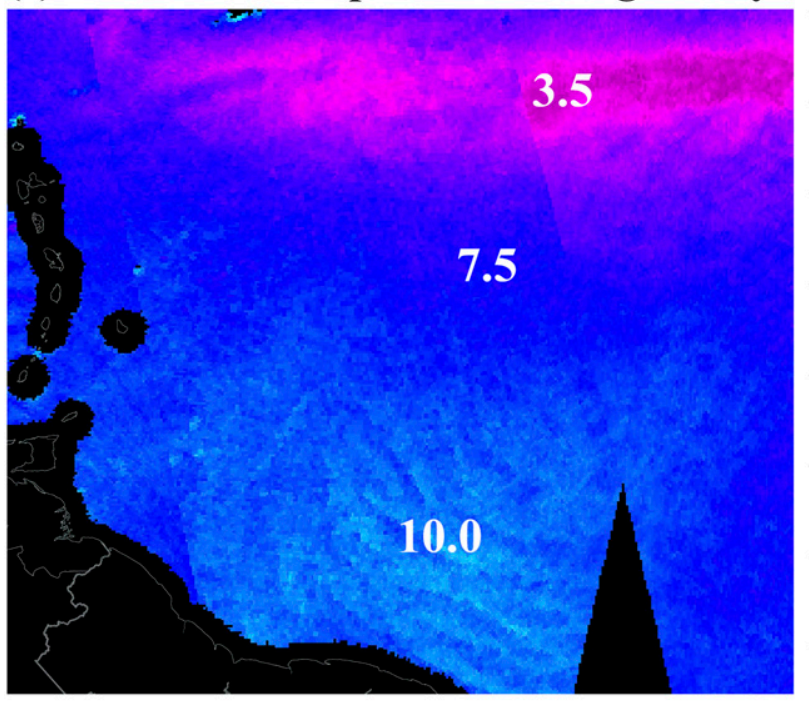

(b) GOES-E IR, Feb 10600 UTC

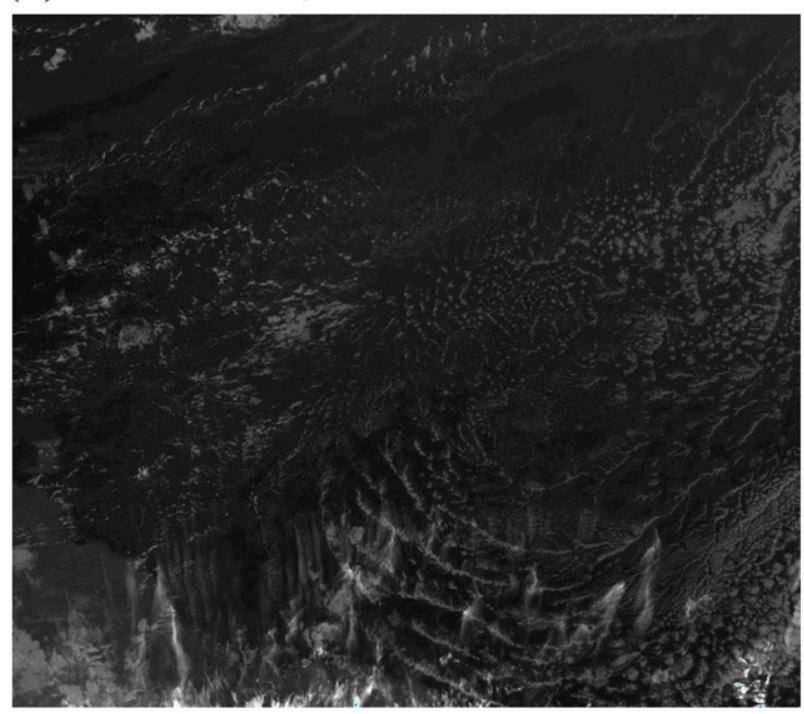

(d) GOES-E cloud tops, Feb 10140 UTC

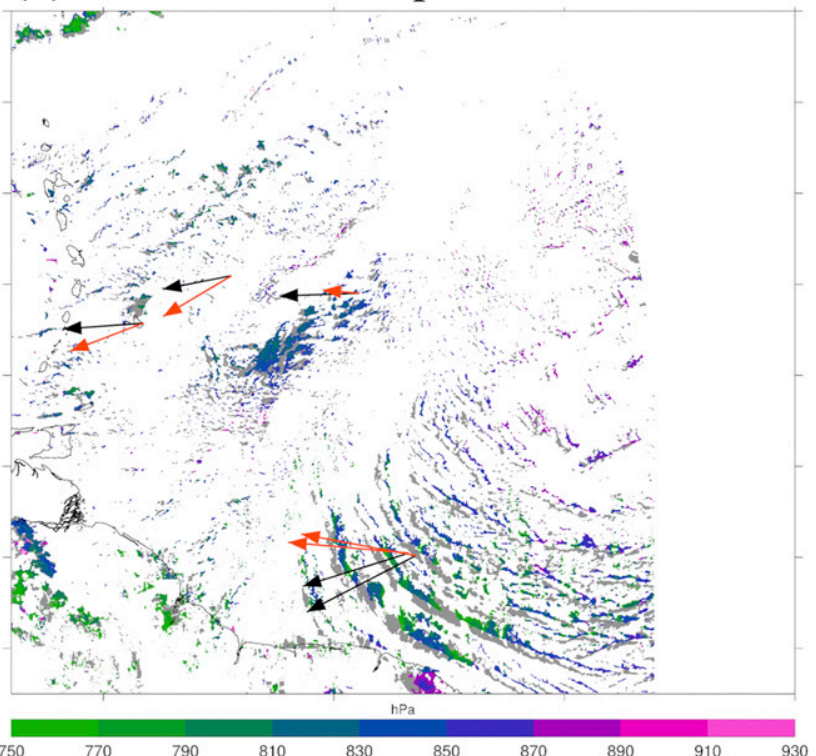

FIG. 1. Cloud lines observed during $31 \mathrm{Jan}$ and $1 \mathrm{Feb} 2020$ over the tropical Atlantic Ocean. All maps show the area $5^{\circ}-20^{\circ} \mathrm{N}, 45^{\circ}-62^{\circ} \mathrm{W}$. (a) Visible $(0.64 \mu \mathrm{m})$ GOES-E image. (b) Infrared (10.3 $\mu \mathrm{m}$ ) GOES-E image. The contrast was enhanced in (a) and (b) after downloading the images from NASA's Worldview application. (c) GCOM-W1 AMSR2 wind speed with typical values of 10-m wind for pink, blue, and azure shadings printed on top $\left(\mathrm{m} \mathrm{s}^{-1}\right)$. The left portion of the image shows the night product, and the right portion shows the day product. (d) GOES-E cloud-top pressure at 0140 UTC (colored shading) and cloud mask 40 min later (0220 UTC; gray shading; no data are available to the east of $49^{\circ} \mathrm{W}$ ). Arrows in (d) show the wind direction averaged below $500 \mathrm{~m}$ (black) and between 1000 and $1500 \mathrm{~m}$ (orange) from radiosondes launched between 0042 and 0253 UTC 1 Feb 2020. Their length is proportional to the wind speed, which is examined quantitatively in Fig. 4, below.

$$
\int_{0}^{h_{0}} d_{0} \rho_{0} d z=\frac{1}{2} \rho_{0} C_{D} u_{0}^{2}=-h_{0} \frac{\partial p_{0}}{\partial x},
$$

where $C_{D}$ is the drag coefficient, which also depends on $u_{0}$. The integral from $z=0$ to $z=h$ of the horizontal momentum balance of the total flow is

$$
h \rho_{0}\left(\frac{\partial}{\partial t}+u \frac{\partial}{\partial x}\right) u+h \frac{\partial p}{\partial x}=-\frac{1}{2} \rho_{0} C_{D} u^{2}
$$

Linearizing this equation and using Eq. (3) results in

$$
i k h_{0} \rho_{0}\left(c-u_{0}\right) u^{\prime}-i k h_{0} p^{\prime}+h^{\prime} \frac{\partial p_{0}}{\partial x}=-\rho_{0} C_{D} u^{\prime} u_{0}
$$




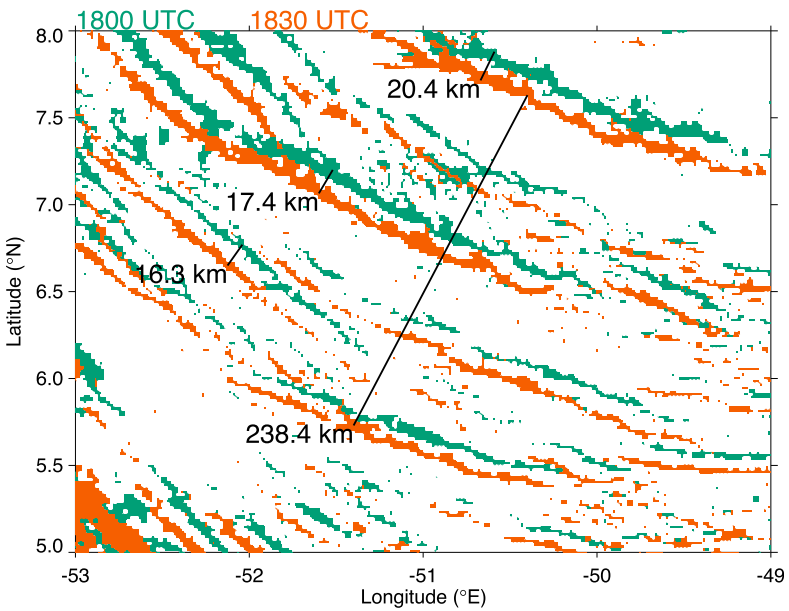

FIG. 2. Cloud lines at 1800 UTC (green) and 1830 UTC (orange) from the GOES-E 10.3- $\mu \mathrm{m}$ infrared band. Colored areas mark brightness temperatures colder than $294.5 \mathrm{~K}$.

We next replace the term $\partial p_{0} / \partial x$ with Eq. (3), take the derivative with respect to $x$, and use Eq. (1) to arrive at

$$
\frac{h^{\prime}}{h_{0}}\left[k h_{0}\left(c-u_{0}\right)^{2}+\frac{3}{2} i C_{D} u_{0}^{2}-i C_{D} u_{0} c\right]=\frac{h_{0} k}{\rho_{0}} p^{\prime} .
$$

At the interface of the well-mixed boundary layer and the overlying layer, pressure and height perturbations need to be continuous. For the overlying layer we assume a constant wind speed $u_{*}$, a constant density $\rho_{*}$, and neutral stability. Vertical velocity perturbations in the upper layer are of the following form:

$$
w_{*}^{\prime}=e^{i(\omega t-k x)-k z} .
$$

The displacement $h_{*}^{\prime}$ in the upper layer has to be

$$
h_{*}^{\prime}=-\frac{i w_{*}^{\prime}}{k\left(c-u_{*}\right)}
$$

to satisfy the definition of the vertical velocity:

$$
w_{*}^{\prime} \equiv \frac{d h_{*}^{\prime}}{d t} \equiv \frac{\partial h_{*}^{\prime}}{\partial t}+u_{*} \frac{\partial h_{*}^{\prime}}{\partial x} .
$$

Similarly, the pressure perturbation in the upper layer has to be

$$
p_{*}^{\prime}=i\left(c-u_{*}\right) \rho_{*} w_{*}^{\prime}
$$

to satisfy the vertical momentum equation:

$$
\frac{d w_{*}^{\prime}}{d t}=-\frac{1}{\rho_{*}} \frac{\partial p_{*}}{\partial z}-g=-\frac{1}{\rho_{*}} \frac{\partial p_{*}^{\prime}}{\partial z} .
$$

Therefore, at the perturbation interface, with $h^{\prime}=h_{*}^{\prime}$ being the displacement relative to $h_{0}$, we can express

$$
p_{*}^{\prime}=-\left(c-u_{*}\right)^{2} \rho_{*} k h^{\prime}+\left(\rho_{0}-\rho_{*}\right) g h^{\prime},
$$

where the extra term $\left(\rho_{0}-\rho_{*}\right) g h^{\prime}$ accounts for the different densities of the layers. By defining the fractional drop in density as $f=\left(\rho_{0}-\rho_{*}\right) / \rho_{0}$ and replacing $p^{\prime}$ with $p_{*}^{\prime}$, Eq. (6) becomes

$$
\begin{aligned}
\frac{1}{k h_{0}}\left[k h_{0}\left(c-u_{0}\right)^{2}+\right. & \left.\frac{3}{2} i C_{D} u_{0}^{2}-i C_{D} u_{0} c\right] \\
& +\left(c-u_{*}\right)^{2} k(1-f) h_{0}-f g h_{0}=0 .
\end{aligned}
$$

With $c=c_{R}+i c_{I}$ we can split Eq. (12) into its real and imaginary parts:

$$
\begin{aligned}
& \left(c_{R}-u_{0}\right)^{2}-c_{I}^{2}+C_{D} \frac{c_{I} u_{0}}{k h_{0}} \\
& +k h_{0}(1-f)\left(c_{R}-u_{*}\right)^{2}-f g h_{0}=0 \quad \text { and }
\end{aligned}
$$

$$
2 c_{I}\left[\left(c_{R}-u_{0}\right)+k h_{0}(1-f)\left(c_{R}-u_{*}\right)\right]=C_{D} \frac{u_{0}}{k h_{0}}\left(c_{R}-\frac{3}{2} u_{0}\right) .
$$
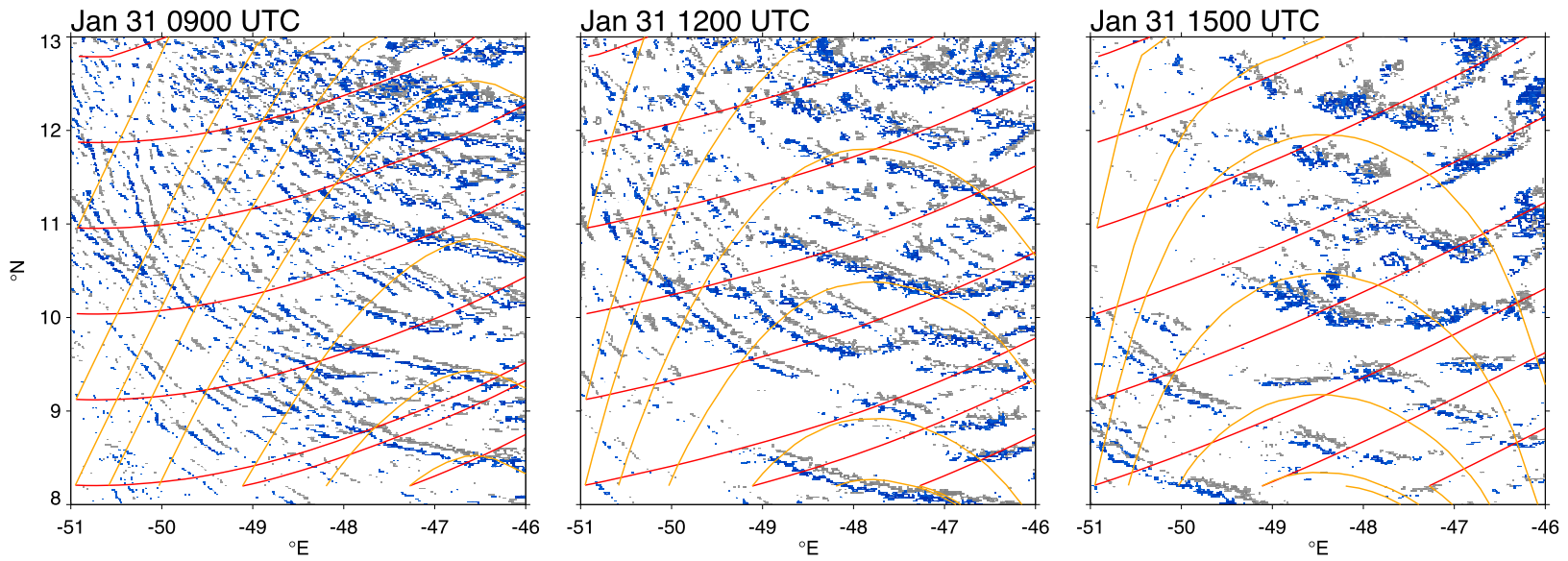

FIG. 3. Cloud lines at the time shown above each panel (gray) and $30 \mathrm{~min}$ later (blue). Colored areas mark brightness temperatures between 290 and $293 \mathrm{~K}$ in the GOES-E 10.3- $\mu \mathrm{m}$ infrared band. Overlaid are streamlines of ERA5 horizontal winds averaged below 500-m altitude (red) and streamlines indicating the shear vector between the surface and $1500 \mathrm{~m}$ (yellow). 
Numerical solutions to these equations are shown in section $4 \mathrm{~b}$. It is still useful to consider the longwave approximation $k h_{0} \ll 1$. Then, Eq. (13b) simplifies to

$$
c_{I}=-\frac{C_{D}}{2} \frac{u_{0}}{k h_{0}} \frac{\left(\frac{3}{2} u_{0}-c_{R}\right)}{\left(c_{R}-u_{0}\right)} .
$$

The growth rate is

$$
v=\frac{1}{\tau}=\frac{1}{w} \frac{d w}{d t} \approx-c_{I} k
$$

Thus, perturbations moving downstream have positive growth rates for $u_{0}<c_{R}<(3 / 2) u_{0}$, as was also discussed by Chimonas (1993).

\section{Case study}

\section{a. Observed cloud field}

A particularly distinct case of arc-shaped cloud lines occurred from 31 January to 1 February 2020 over the western tropical Atlantic (Figs. 1a,b). We select this event for a case study because it coincided with the EUREC ${ }^{4} \mathrm{~A}$ field campaign and was located in an area with radiosonde launches. The first lines appeared around 0600 UTC 31 January and a clear pattern is seen at 1800 UTC 31 January. Compared to the lines at 1800 UTC 31 January, those at 0600 UTC 1 February are sharper. We interpret this change in morphology as an indication of a growing instability. The line spacing in Figs. $1 \mathrm{a}$ and $1 \mathrm{~b}$ is about $60 \mathrm{~km}$, as is evident from Fig. 2. In Fig. 1a and on the edges of the pattern in Fig. $1 \mathrm{~b}$ the spacing is about $40 \mathrm{~km}$. Cloud tops are found at around 870-790 hPa (Fig. 1d), corresponding to about $1-2 \mathrm{~km}$.

The synoptic conditions on 31 January and 1 February 2020 were characterized by a ridge at $18^{\circ}-19^{\circ} \mathrm{N}$, which is marked by low wind speeds (pink colors) in Fig. 1c. The cloud lines occurred to the southeast of the ridge in a location where wind speeds vary between 8 and $11 \mathrm{~m} \mathrm{~s}^{-1}$. On average, this would correspond to $u_{0}=9.5 \mathrm{~m} \mathrm{~s}^{-1}$. Figures $1 \mathrm{~d}$ and 2 provide evidence that the cloud lines are moving along the near-surface $(0-500 \mathrm{~m})$ wind direction at a speed of about $9.1-11.3 \mathrm{~m} \mathrm{~s}^{-1}$. On average, this would correspond to $C_{R}=10.2 \mathrm{~m} \mathrm{~s}^{-1}$. Hence, the inequalities $u_{0}<c_{R}<(3 / 2) u_{0}$ hold on average [Eq. (14)]: the cloud lines are moving at phase speeds that are unstable.

Figure 3 focuses on the early development stage of the cloud bands. The cloud bands form approximately perpendicular to the near surface wind, which at 0900 UTC and between $-46^{\circ}$ and $-51^{\circ} \mathrm{E}$ changes from west-southwestward to westward. While this directional change may contribute to the curvature of the bands, they appear to have a stronger curvature than the nearsurface streamlines. This morphology could point to the existence of a radially propagating initial instability. Figure 3 also shows the streamlines that correspond to the shear vector across the cloud layer. The alignment of the shear vector relative to the cloud bands and to the near-surface wind vector varies, which suggests that shear is not likely to play a role for the formation of these cloud bands.

Cloud patterns of this type occur frequently over the tropical Atlantic. We browsed satellite images at a fixed time (1600 UTC) for each day of January 2020 and found arc-shaped banded clouds
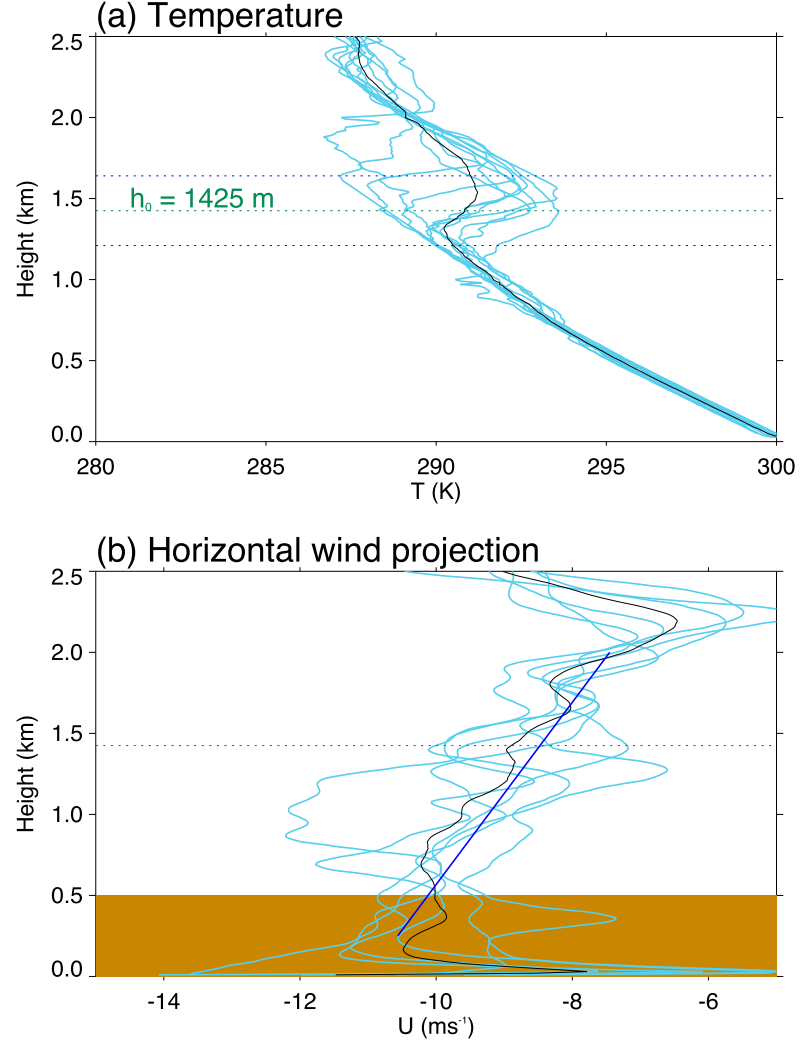

(c) Potential temperature

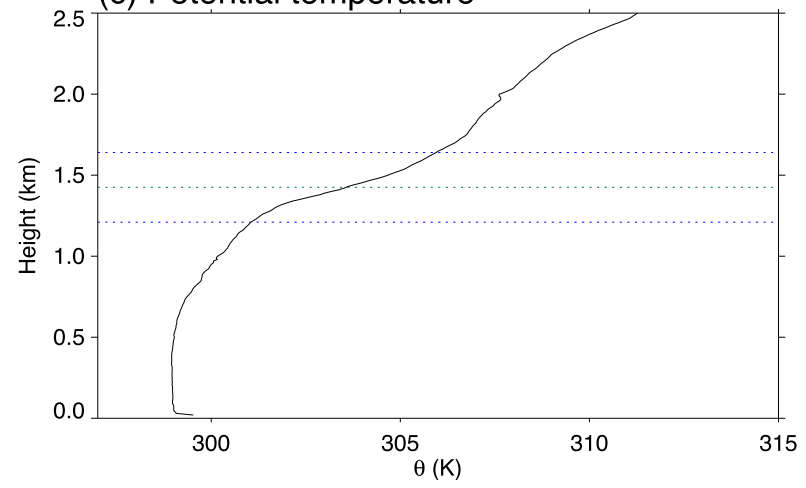

FIG. 4. Data from ascending and descending radiosonde soundings inside $7.7^{\circ}-8.1^{\circ} \mathrm{N}, 53.1^{\circ}-53.7^{\circ} \mathrm{W}$ launched between 1800 UTC 31 Jan and 0600 UTC 1 Feb 2020. (a) Temperature from all soundings, with $h_{0}$ marked by the horizontal green dashed line. The horizontal blue dashed lines mark the inversion layer and are spaced $430 \mathrm{~m}$ apart. (b) Wind projected against the $0-500-\mathrm{m}$ wind direction from ascending soundings. The dark-blue line is the result of a linear regression between 250 and $2000 \mathrm{~m}$. (c) Average potential temperature profile (black) from ascending and descending soundings.

on 14 days inside the region $9^{\circ} \mathrm{S}-15^{\circ} \mathrm{N}, 80^{\circ}-5^{\circ} \mathrm{W}$. GCOM-W1 wind speeds ranged between 7 and $10 \mathrm{~m} \mathrm{~s}^{-1}$ for all of these cases.

\section{b. Comparison with theoretical solutions}

To arrive at the theoretical solutions to the drag instability [Eqs. (13a) and (13b)], we solve this coupled-equation system 

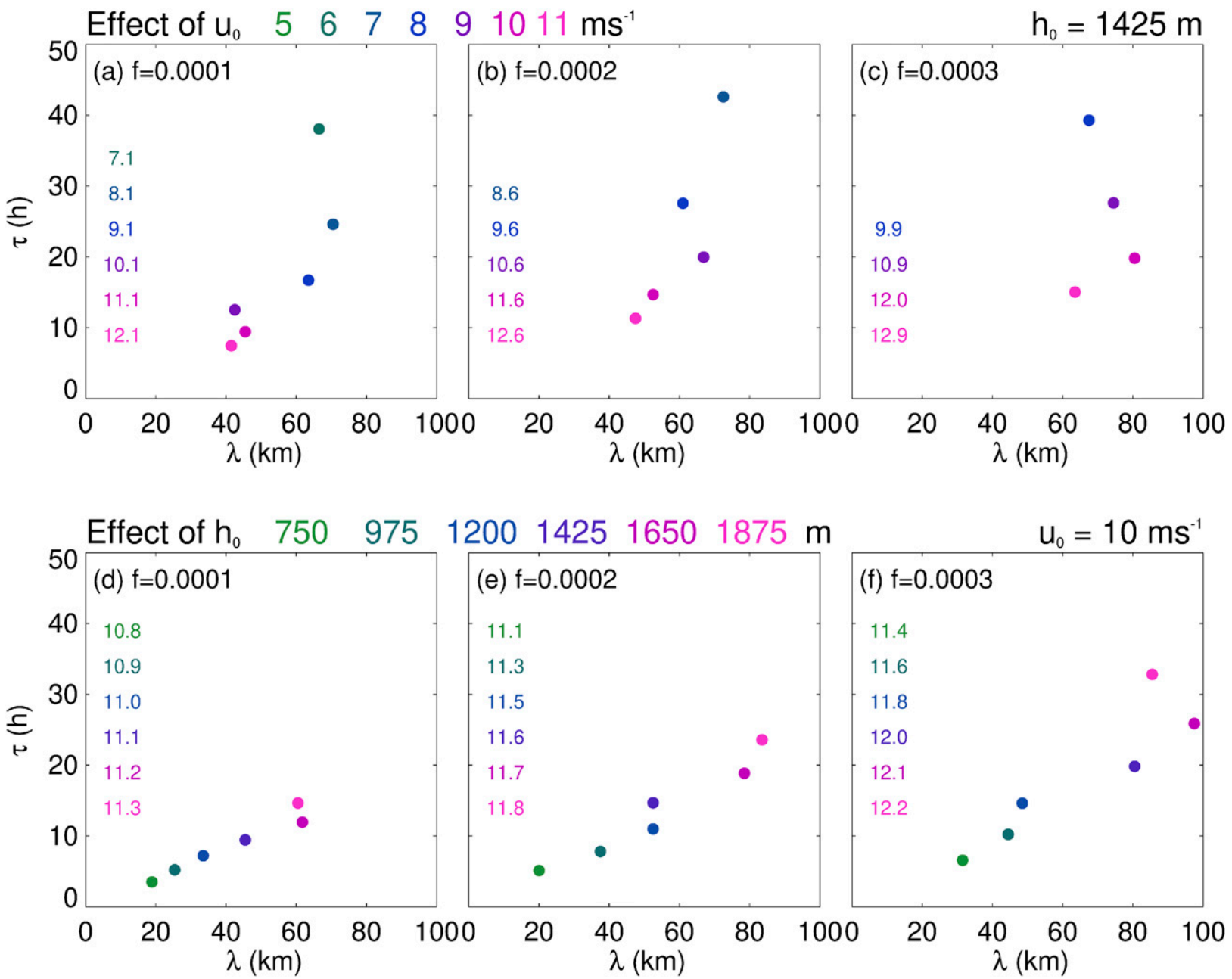

FIG. 5. Numerical solution to Eq. (13). Shown is doubling time vs horizontal wavelength for (left) $f=0.0001$, (center) $f=0.0002$, and (right) $f=0.0003$ for (a)-(c) the effect of changes in $u_{0}$ at constant $h_{0}=1425 \mathrm{~m}$ and (d)-(f) the effect of changes in $h_{0}$ at constant $u_{0}=$ $10 \mathrm{~m} \mathrm{~s}^{-1}$. Filled circles mark the minimum of $\tau$. The corresponding phase velocities are listed in each panel.

numerically. For each computation, $h_{0}, u_{0}\left(=u_{*}\right), C_{D}$, and $f$ are fixed such that the two equations describe the nonlinear relationship between $k, C_{I}$, and $C_{R}$. We then vary $C_{R}$ between $u_{0}$ and $(3 / 2) u_{0}$, because this corresponds to unstable solutions. For each value of $C_{R}$, we now have two equations for the two unknown variables $C_{I}$ and $k$. Thus, by varying $C_{R}$, we obtain the function $C_{I}(k)$. The minimum of this function corresponds to the wavenumber with the fastest growth rate. This value of $k$ and the associated values of $C_{I}$ and $C_{R}$, respectively, are saved and are shown in Figs. 5 and 6.

We estimate the parameters $u_{0}, f$, and $h_{0}$ in Eqs. (13a) and (13b) from radiosonde soundings near the location of the southernmost arrows in Fig. 1d. The average inversion height is at $h_{0}=1425 \mathrm{~m}$ (Fig. 4a), which is consistent with the observed cloud depth (Fig. 1d). To estimate the horizontal wind speed we project the wind at all altitudes against the direction of the $0-500-\mathrm{m}$ averaged wind (Fig. 4b), because this is the direction in which the cloud lines are moving (Fig. 1d). The mean wind speed at $0-500 \mathrm{~m}$ is $9.96 \mathrm{~m} \mathrm{~s}^{-1}$. As laid out in section 3 , the equations assume that $u_{0}$ is constant below $h_{0}$ while $u_{*}$ is constant above $h_{0}$. Of course, such conditions are not met in reality. The average wind profile between 250 and $2000 \mathrm{~m}$ can be approximated as $u(z)=-11.0 \mathrm{~m} \mathrm{~s}^{-1}+$ $1.6 \mathrm{z} \mathrm{m} \mathrm{s}^{-1} \mathrm{~km}^{-1}$ (Fig. 4b). The solutions to Eqs. (13a) and (13b) are computed for $u_{0}$ in the interval 5-11 $\mathrm{m} \mathrm{s}^{-1}$, which encompasses the observed variability. Moreover, we use the approximation $u_{*}=u_{0}$ for all computations.

Last, we need to estimate the parameter $f$, which is the fractional drop in density from below $h_{0}$ to above $h_{0}$. While it is common to describe atmospheric boundary layer processes in two-layer constant-density frameworks, density is a continuous function of height, so that $f$ could in principle be arbitrarily small. Physically, $f$ represents the density change that waves of amplitude $h^{\prime}$ experience at level $h_{0}$. Therefore, to estimate $f$, we compute perturbations $\theta^{\prime}(z)$ from a 100 -m running mean $\bar{\theta}(z)$, because these perturbations are likely to result from gravity wave-induced motions, even though they are not necessarily the waves that drive the cloud patterns. At each altitude, $f(z)=\theta^{\prime}(z) / \bar{\theta}(z)$. When averaged around the inversion level 


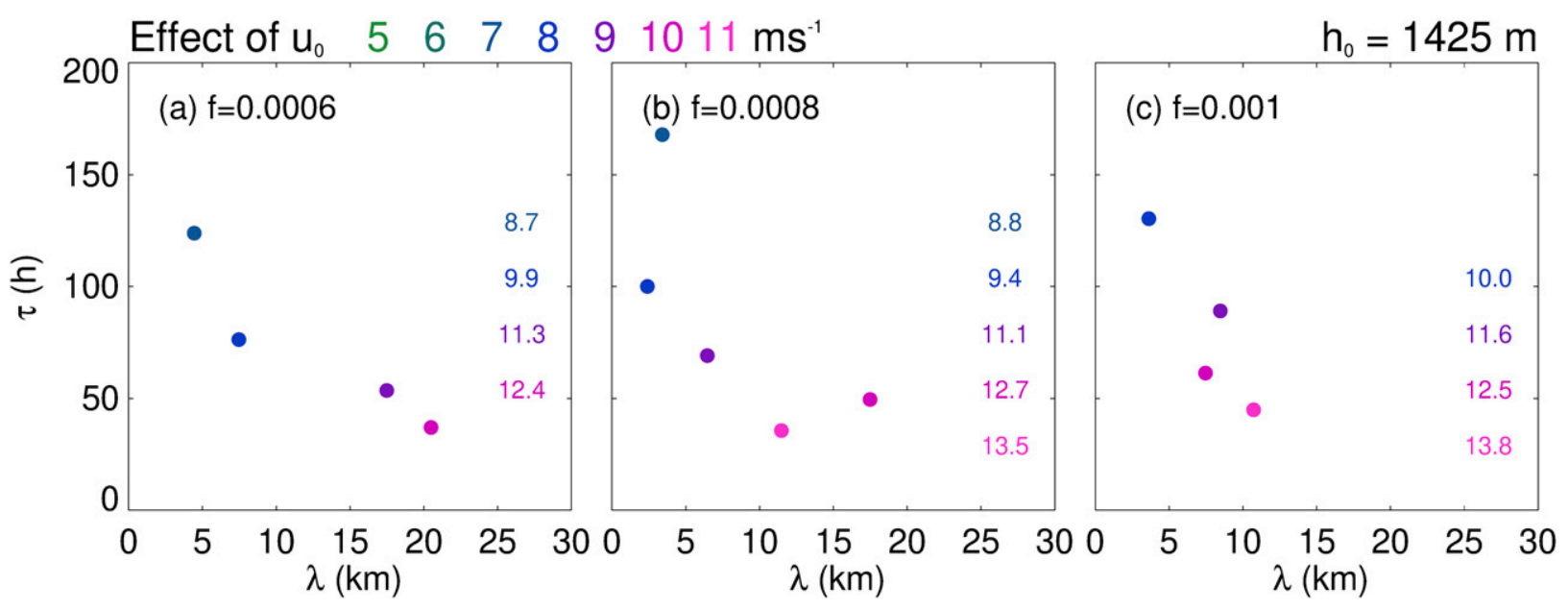

FIG. 6. As in Figs. 5a-c, but for (a) $f=0.0006$, (b) $f=0.0008$, and (c) $f=0.001$.

(dark-blue horizontal dashed lines) in Fig. 4c, we obtain $f=0.00025$. (The calculation was repeated with a $1000-\mathrm{m}$ running mean, which also gives $f=0.00025)$. To test the sensitivity to $f$, we explore values for $f$ of $0.0001,0.0002$, and 0.0003 in our computation.

Figure 5 shows how $\tau$, the inverse growth rate [Eq. (15)], varies with the horizontal wavelength $\lambda$. We derive the surface drag coefficient from the roughness Reynolds number $R_{r}$, which Fairall et al. (1996) reported as approximately $(0.4,0.7$, 1.0, 1.7, 2.0, 3.0, 4.0) for 10-m wind speeds of $(5,6,7,8,9,10$, 11) $\mathrm{m} \mathrm{s}^{-1}$ above the ocean. SethuRaman and Raynor (1975) defined these values of $R_{r}$ as a moderately rough regime, where $C_{D}$ can be computed as $2 \times\left(0.42 \log R_{r}+1.23\right) \times 10^{-3}$ [multiplying by 2 accounts for the difference in our definition of $C_{D}$; Eq. (3)]. This results in values of $C_{D}=(0.0021,0.0023,0.0025$, $0.0026,0.0027,0.0028,0.0030)$ at the above $R_{r}$ values.

The time $\tau$ decreases with increasing wind speed, whereas $\tau$ and $\lambda$ increase with increasing $f$ (Figs. 5a-c). For $u=10 \mathrm{~m} \mathrm{~s}^{-1}$ and $h_{0}=1425 \mathrm{~m}, \lambda$ equals $55-85 \mathrm{~km}(f=0.0002, f=0.0003)$. When compared with the observed cloud spacing $(60 \pm 20 \mathrm{~km})$ this is the correct order of magnitude. Amplitudes would double after $15-20 \mathrm{~h}$. This time is also consistent with observations and the evolution between 1800 UTC 31 January and 0600 UTC 1 February (Figs. 1a,b). For slow wind speeds $u_{0}<6 \mathrm{~m} \mathrm{~s}^{-1}, \tau$ is far more than a day. These results are consistent with the fact that all occurrences of arc-shaped cloud lines in observations in January 2020 were accompanied by $10-\mathrm{m}$ wind speeds of $7-10 \mathrm{~m} \mathrm{~s}^{-1}$. Deeper mixed layers produce larger $\lambda$ (Figs. $5 \mathrm{~d}-\mathrm{f}$ ). For $10 \mathrm{~m} \mathrm{~s}^{-1}$ and $h_{0}=1425 \mathrm{~m}, \lambda$ corresponds well to the observations.

The results also suggest that $f$ is not likely to be much greater than our estimated value of 0.00025 . Nevertheless, to gain a deeper understanding of how $f$ affects the solutions, Fig. 6 repeats Fig. 5 for $f \in(0.0006,0.0008,0.001)$. It is interesting to note that, for these larger values of $f$, wavelengths increase with increasing wind speed. Overall, however, the wavelengths in Fig. 6 are much smaller than in Fig. 5 and the doubling times are multiple days. Small $f$, as estimated from the soundings, implies that only small vertical displacements may be needed to trigger an initial instability, rather than displacements of several hundreds of meters. This seems plausible given that the cloud patterns occur frequently.

\section{Conclusions}

This study adds a new cloud pattern to the list of shallow convective cloud patterns that have an accompanying theory to describe their characteristic properties. We studied a case of widespread arc-shaped cloud lines over the tropical Atlantic Ocean that occurred during the EUREC ${ }^{4} \mathrm{~A}$ field campaign. The availability of radiosonde soundings allowed us to determine parameters, such as the mixed-layer depth and mean wind speed, that are required for solving the equation system of drag-instability waves. The drag-instability mechanism was originally proposed by Jeffreys (1925) and adapted for the atmospheric boundary layer by Chimonas (1993). A central assumption of the mechanism is that the aerodynamic surface friction is approximately proportional to the square of the wind speed and is felt by the full depth of the boundary layer. Neither this, nor previous studies validated this assumption, although Chimonas (1993) and Chimonas (1994) provide arguments for its plausibility. Until now, the drag instability had not been considered as an explanation for shallow cloud lines that form at large oblique angles to the near-surface wind. Our results suggest that these patterns can form over the course of approximately 0.5 day when the mixed layer is about $1 \mathrm{~km}$ deep and the mean wind speed in this layer is greater than $7 \mathrm{~ms}^{-1}$. Under such conditions, wavelike perturbations of the horizontal wind speed are able to modulate the surface friction in a way to reinforce themselves. A strong subtropical high, as it was present in the case we presented, is one synoptic condition that can sustain these relatively strong wind speeds over a long time and a large area. We solved the drag-instability equations and compared the predicted growth rate and wavelength with their observed counterparts. The theoretical horizontal wavelengths of 40$80 \mathrm{~km}$ are in good agreement with observations $(60 \pm 20 \mathrm{~km})$, and a theoretical doubling of the amplitudes within $15-20 \mathrm{~h}$ is also consistent with observations. 
Acknowledgments. Author Stephan was supported by the Minerva Fast Track Program of the Max Planck Society. She thanks Hauke Schulz for help with the download of GOES-E satellite data.

Data availability statement. Visible $(0.64 \mu \mathrm{m}$; Band 2) and infrared (10.3 $\mu \mathrm{m}$, Band 13) images of the GOES-E Advanced Baseline Imager (ABI; Schmit et al. 2017) are provided by NASA's Worldview application (https://worldview.earthdata.nasa.gov). The GCOM-W1 AMSR2 data are available online from the NASA Global Hydrology Resource Center DAAC, Huntsville, Alabama, and can be browsed in NASA's Worldview application (https://worldview.earthdata.nasa.gov). The Langley SATCORPS group of NASA is the source of the GOES-E cloud-top pressure product, which is only available for the focus region of the Atlantic Tradewind Ocean-Atmosphere Mesoscale Interaction Campaign (ATOMIC)/ Ocean-Atmosphere component of EUREC ${ }^{4} \mathrm{~A}$. Radiosonde data of EUREC ${ }^{4} \mathrm{~A}$ are published online (https://doi.org/10.25326/62). The ICON source code is publicly available. The ERA5 reanalysis (ERA5 2017) is publicly available from the ECMWF.

\section{REFERENCES}

Agee, E. M., 1987: Mesoscale cellular convection over the oceans. Dyn. Atmos. Oceans, 10, 317-341, https://doi.org/10.1016/ 0377-0265(87)90023-6.

Atkinson, B. W., and J. Wu Zhang, 1996: Mesoscale shallow convection in the atmosphere. Rev. Geophys., 34, 403-431, https:// doi.org/10.1029/96RG02623.

Balaji, V., and T. L. Clark, 1988: Scale selection in locally forced convective fields and the initiation of deep cumulus. J. Atmos. Sci., 45, 3188-3211, https://doi.org/10.1175/1520-0469(1988) 045<3188:SSILFC > 2.0.CO;2.

Brown, R. A., 1980: Longitudinal instabilities and secondary flows in the planetary boundary layer: A review. Rev. Geophys., 18, 683-697, https://doi.org/10.1029/RG018i003p00683.

Chimonas, G., 1993: Surface drag instabilities in the atmospheric boundary layer. J. Atmos. Sci., 50, 1914-1924, https://doi.org/ 10.1175/1520-0469(1993)050<1914:SDIITA > 2.0.CO;2.

_ 1994: Jeffreys' drag instability applied to waves in the lower atmosphere: Linear and nonlinear growth rates. J. Atmos. Sci., 51, 3758-3775, https://doi.org/10.1175/1520-0469(1994)051<3758: JDIATW $>2.0 . \mathrm{CO} ; 2$.

Clark, T. L., T. Hauf, and J. P. Kuettner, 1986: Convectively forced internal gravity waves: Results from two-dimensional numerical experiments. Quart. J. Roy. Meteor. Soc., 112, 899925, https://doi.org/10.1002/qj.49711247402.

ERA5, 2017: ERA5: Fifth generation of ECMWF atmospheric reanalyses of the global climate. Copernicus Climate Change Service Climate Data Store (CDS), accessed 29 November 2020, https://cds.climate.copernicus.eu/cdsapp\#!/home.

Fairall, C. W., E. F. Bradley, D. P. Rogers, J. B. Edson, and G. S. Young, 1996: Bulk parameterization of air-sea fluxes for tropical ocean-global atmosphere coupled-ocean atmosphere response experiment. J. Geophys. Res., 101,3747-3764, https:// doi.org/10.1029/95JC03205.

Hauf, T., and T. L. Clark, 1989: Three-dimensional numerical experiments on convectively forced internal gravity waves. Quart. J. Roy. Meteor. Soc., 115, 309-333, https://doi.org/ 10.1002/qj.49711548606.

Jeffreys, H., 1925: On fluid motions produced by differences of temperature and humidity. Quart. J. Roy. Meteor. Soc., 51, 347-356, https://doi.org/10.1002/qj.49705121604.

Khanna, S., and J. G. Brasseur, 1998: Three-dimensional buoyancyand shear-induced local structure of the atmospheric boundary layer. J. Atmos. Sci., 55, 710-743, https://doi.org/10.1175/ 1520-0469(1998)055<0710:TDBASI >2.0.CO;2.

Kummerow, C., R. Ferraro, and D. Duncan, 2015: NRT AMSR2 L2B global swath GSFC profiling algorithm 2010: Surface precipitation, wind speed over ocean, water vapor over ocean and cloud liquid water over ocean. NASA Global Hydrology Center DAAC, accessed 26 June 2020, https://doi.org/ 10.5067/AMSR2/A2_RainOcn_NRT.

Lane, T. P., and T. L. Clark, 2002: Gravity waves generated by the dry convective boundary layer: Two-dimensional scale selection and boundary-layer feedback. Quart. J. Roy. Meteor. Soc., 128, 1543-1570, https://doi.org/10.1002/qj.200212858308.

Moeng, C.-H., and P. P. Sullivan, 1994: A comparison of shear- and buoyancy-driven planetary boundary layer flows. J. Atmos. Sci., 51, 999-1022, https://doi.org/10.1175/1520-0469(1994) 051<0999:ACOSAB >2.0.CO;2.

Nicholls, S. D., and G. S. Young, 2007: Dendritic patterns in tropical cumulus: An observational analysis. Mon. Wea. Rev., 135, 1994-2005, https://doi.org/10.1175/MWR3379.1.

NOAA, 2018: NOAA GOES-R series advanced baseline imager (ABI) level 2 cloud top height (ACHA). GOES-R algorithm working group, and GOES-R series program office, NOAA National Centers For Environmental Information, accessed 26 June 2020, https://doi.org/10.7289/V5HX19ZQ.

Schmit, T. J., P. Griffith, M. M. Gunshor, J. M. Daniels, S. J. Goodman, and W. J. Lebair, 2017: A closer look at the ABI on the GOES-R series. Bull. Amer. Meteor. Soc., 98, 681-698, https://doi.org/10.1175/BAMS-D-15-00230.1.

SethuRaman, S., and G. S. Raynor, 1975: Surface drag coefficient dependence on the aerodynamic roughness of the sea. J. Geophys. Res., 80, 4983-4988, https://doi.org/10.1029/ JC080i036p04983.

Stephan, C. C., and Coauthors, 2020: Ship- and island-based atmospheric soundings from the 2020 EUREC $^{4}$ A field campaign. Earth Syst. Sci. Data, https://doi.org/10.5194/ essd-2020-174, in press.

Stevens, B., and Coauthors, 2020: Sugar, gravel, fish and flowers: Mesoscale cloud patterns in the trade winds. Quart. J. Roy. Meteor. Soc., 146, 141-152, https://doi.org/ 10.1002/qj.3662.

Young, G. S., D. A. R. Kristovich, M. R. Hjelmfelt, and R. C. Foster, 2002: Rolls, streets, waves, and more: A review of quasi-two-dimensional structures in the atmospheric boundary layer. Bull. Amer. Meteor. Soc., 83, 997-1002, https://doi.org/ 10.1175/1520-0477(2002)083<0997:RSWAMA>2.3.CO;2. 